D. D. BAINOV (Sofia)

M. B. Dimitrova (Sliven)

\title{
SUFFICIENT CONDITIONS FOR OSCILLATION AND NONOSCILLATION OF THE SOLUTIONS OF OPERATOR-DIFFERENTIAL EQUATIONS WITH PIECEWISE CONSTANT ARGUMENT
}

Abstract. Effective sufficient conditions for oscillation and nonoscillation of solutions of some operator-differential equations with piecewise constant argument are found.

1. Introduction. In [1] sufficient conditions are obtained for oscillation of all solutions of the operator-differential equation with piecewise constant argument

$$
y^{\prime}(t)+q(t) y(t)+p(t) y([t])=0,
$$

where $p, q \in C([0, \infty) ; \mathbb{R})$ and $\lim _{t \rightarrow \infty} p(t)=\lim _{t \rightarrow \infty} q(t)=\infty$.

Some mathematical models in biology [3] are described by means of equations of the form (1).

In [5] sufficient conditions are obtained for oscillation and nonoscillation of solutions of the equations

$$
\begin{aligned}
& y^{\prime}(t)+p(t) f(y([t]))=0, \\
& y^{\prime}(t)+p(t) f(y([t]))=h(t) .
\end{aligned}
$$

In the present paper the operator-differential equations with piecewise constant argument

$$
\begin{aligned}
& x^{\prime}(t)+p(t)(\mathcal{A} x)([t])=0, \\
& x^{\prime}(t)+p(t)(\mathcal{A} x)([t])=h(t)
\end{aligned}
$$

1991 Mathematics Subject Classification: Primary 34K15; Secondary 34L30.

Key words and phrases: oscillation and nonoscillation, operator-differential equations, piecewise constant argument. 
are investigated, where $\mathcal{A}$ is an operator with certain properties. Sufficient conditions for oscillation and nonoscillation of solutions of equations (2) and (3) are obtained. Some particular realizations of the operator $\mathcal{A}$ are considered.

2. Preliminaries. Consider the operator-differential equations

$$
\begin{aligned}
& x^{\prime}(t)+p(t)(\mathcal{A} x)([t])=0, \\
& x^{\prime}(t)+p(t)(\mathcal{A} x)([t])=h(t),
\end{aligned}
$$

where $\mathcal{A}$ is an operator and $p(\cdot)$ is locally integrable function in $\mathbb{R}$. Let $t_{0}$ be a fixed real number. Denote by $C\left(\left[t_{0}, \infty\right) ; \mathbb{R}\right)$ the set of all continuous functions $u:\left[t_{0}, \infty\right) \rightarrow \mathbb{R}$, and by $L_{\mathrm{loc}}\left(\left[t_{0}, \infty\right) ; \mathbb{R}\right)$ the set of all functions $u:\left[t_{0}, \infty\right) \rightarrow \mathbb{R}$ which are Lebesgue integrable in each compact subinterval of $\left[t_{0}, \infty\right)$.

Definition 1. By a solution of equation (3) in the interval $\left[t_{0}, \infty\right)$ we mean any function $x(t)$ satisfying the following conditions:

1. $x \in C\left(\left[t_{0}, \infty\right) ; \mathbb{R}\right)$.

2. The derivative $x^{\prime}(t)$ exists at any point $t \geq t_{0}$ with the possible exception of the integer values of $t$, at which the right-hand derivative exists.

3. The function $x(t)$ satisfies equation (3) in each finite interval $[n, n+1)$ $\subset\left[t_{0}, \infty\right)$, where $n \geq t_{0}$ and $n$ is an integer.

The set of all functions satisfying conditions 1 and 2 of Definition 1 will be denoted by $\mathcal{D}_{t_{0}}$.

DEFinition 2. A solution $x(t)$ of the equation (3) is said to be regular if $\sup \{x(t): t \geq T\}>0$ for $T \geq N_{x}$, where $N_{x} \geq t_{0}$ is an integer.

Definition 3. A regular solution $x(t)$ of the equation (3) is said to oscillate if there exists a sequence $\left\{t_{n}\right\}_{n=1}^{\infty}$ of points such that $\lim _{n \rightarrow \infty} t_{n}=\infty$ and $x\left(t_{n}\right)=0$.

Otherwise the regular solution $x(t)$ is said to be nonoscillating.

Definition 4. A function $u:\left[t_{0}, \infty\right) \rightarrow \mathbb{R}$ is said to eventually enjoy a property $P$ if there exists a point $t_{P, u} \geq t_{0}$ such that for $t \geq t_{P, u}$ it enjoys the property $P$.

We introduce the following conditions:

H1. $p \in L_{\text {loc }}\left(\left[t_{0}, \infty\right) ; \mathbb{R}\right)$, meas $\{s \geq t: p(s) \neq 0\}>0$.

H2. $\mathcal{A}: \mathcal{D}_{t_{0}} \rightarrow L_{\mathrm{loc}}\left(\left[t_{0}, \infty\right) ; \mathbb{R}\right)$.

H3. If $u \in \mathcal{D}_{t_{0}}$ and $u(t) \equiv 0$ eventually, then $(\mathcal{A} u)(t) \equiv 0$ eventually.

H4. If $u \in \mathcal{D}_{t_{0}}$ is eventually nonzero and of constant sign, then so is $\mathcal{A} u$, and they are of the same sign. 


\section{Main results}

THEOREM 1. Let the following conditions hold:

1. Conditions $\mathrm{H} 1-\mathrm{H} 4$ are satisfied.

2. $p(t) \leq 0$ for $t \in\left[t_{0}, \infty\right)$.

Then all regular solutions of the equation (2) are nonoscillating.

Pr o o f. Let $x(t)$ be a regular solution of (2) in $\left[N_{x}, \infty\right)$, where $N_{x} \geq t_{0}$ is an integer. Suppose that there exists an integer $n \geq N_{x}$ such that $x(n)=0$. From (2) it follows that $x^{\prime}(t)=-p(t)(\mathcal{A} x)(n)$ for $t \in[n, n+1)$. Then $x^{\prime}(t)=0$ for $t \in[n, n+1)$, i.e., $x(t)=$ const for $n \leq t<n+1$. Hence if $x(n)=0$ for any integer $n \geq N_{x}$, then by continuity of $x(t), x(t) \equiv 0$ in $[n, \infty)$, which contradicts the requirement that $x(t)$ be a regular solution of (2). Hence there exists an integer $m \geq N_{x}$ such that $x(m) \neq 0$. Let $x(m)>0$ (the case $x(m)<0$ is analogous). Then

$$
x^{\prime}(t)=-p(t)(\mathcal{A} x)(m) \geq 0
$$

for $t \in[m, m+1)$, and so $0<x(m) \leq x(t) \leq x(m+1)$.

Analogously, we obtain $x(m+2)>0$, etc. Hence $x(t)>0$ for $t \geq m$.

THEOREM 2. Let the following conditions hold:

1. Conditions $\mathrm{H} 1-\mathrm{H} 4$ are satisfied.

2. $p(t) \geq 0$ for $t \geq t_{0}$ and

$$
\limsup _{n \rightarrow \infty} \int_{n}^{n+1} p(t) d t<1 \quad \text { for } n \text { integer, } n \geq t_{0} .
$$

3. $(\mathcal{A} u)(t) \leq u(t)$ for any integer $t \in\left[t_{0}, \infty\right)$ and any $u \in \mathcal{D}_{t_{0}}$.

Then all regular solutions of the equation (2) are nonoscillating.

Proof. Let $x(t)$ be a regular solution of $(2)$ in $\left[N_{x}, \infty\right)$, where $N_{x} \geq t_{0}$ is an integer. There exists an integer $n_{1} \geq N_{x}$ and a number $\varepsilon, 0<\varepsilon<1$, such that for $n \geq n_{1}$,

$$
\int_{n}^{n+1} p(t) d t<1-\varepsilon .
$$

As in the proof of Theorem 1 we conclude that there exists an integer $n_{2} \geq n_{1}$ such that $x\left(n_{2}\right) \neq 0$. Let $x\left(n_{2}\right)>0$ (the case $x\left(n_{2}\right)<0$ is analogous).

Integrate (2) from $n_{2}$ to $t$ for $t \in\left[n_{2}, n_{2}+1\right)$ to obtain

$$
x(t)=x\left(n_{2}\right)-\int_{n_{2}}^{t} p(s)(\mathcal{A} x)([s]) d s
$$




$$
\begin{aligned}
& \geq x\left(n_{2}\right)-(\mathcal{A} x)\left(n_{2}\right) \int_{n_{2}}^{n_{2}+1} p(s) d s \\
& \geq(\mathcal{A} x)\left(n_{2}\right)\left[1-\int_{n_{2}}^{n_{2}+1} p(s) d s\right]>0 .
\end{aligned}
$$

Repeating this process, we conclude that $x(t)>0$ for $t \in\left[n_{2}+1, n_{2}+2\right)$, etc., i.e., $x(t)>0$ for $t \geq n_{2}$.

THEOREM 3. Let the following conditions hold:

1. Conditions $\mathrm{H} 1-\mathrm{H} 4$ and condition 3 of Theorem 2 are satisfied.

2. $p(t) \geq 0$ for $t \geq t_{0}$ and

$$
\lim _{n \rightarrow \infty} \int_{n}^{n+1} p(t) d t=0 \quad \text { for } n \text { integer. }
$$

Then each bounded solution of the equation (2) is nonoscillating.

The proof of Theorem 3 follows the scheme of the proof of Theorem 2 .

THEOREM 4. Let the following conditions hold:

1. Condition $\mathrm{H} 2$ is satisfied.

2. $p \in C\left(\left[t_{0}, \infty\right) ; \mathbb{R}\right)$.

3. $h \in C\left(\left[t_{0}, \infty\right) ; \mathbb{R}\right)$.

4. $\lim _{t \rightarrow \infty} h(t) / p(t)=\infty$.

5. If $u \in \mathcal{D}_{t_{0}}$ is eventually nonzero and bounded, then so is $\mathcal{A} u$.

Then all bounded regular solutions of the equation (3) are nonoscillating.

P r o of. Let $x(t)$ be a bounded regular solution of $(3)$ in $\left[N_{x}, \infty\right)$, where $N_{x} \geq t_{0}$ is an integer, i.e., there exists a constant $M_{1}>0$ such that $|x(t)| \leq$ $M_{1}$ for $t \geq N_{x}$. From condition 5 it follows that there exists a constant $M_{2}>0$ and a number $t_{1} \geq N_{x}$ such that $|(\mathcal{A} x)(t)| \leq M_{2}$ for $t \geq t_{1}$. By condition 4 , there exists $T \geq t_{1}$ such that $h(t) \geq M_{2} p(t)$ for $t \geq T$.

Suppose that there exists a sequence $\left\{t_{n}\right\}_{n=1}^{\infty}$ of zeros of $x(t)$ such that $\lim _{n \rightarrow \infty} t_{n}=\infty$. Denote by $t_{k}, t_{k+1}$ two consecutive zeros of $x(t)$ such that $T \leq t_{k} \leq t_{k+1}$.

Integrate (3) from $t_{k}$ to $t_{k+1}$ and obtain

$$
0=\int_{t_{k}}^{t_{k+1}}[h(s)-p(s)(\mathcal{A} x)([s])] d s \geq \int_{t_{k}}^{t_{k+1}}\left[h(s)-M_{2} p(s)\right] d s>0 .
$$

THEOREM 5. Let the following conditions hold:

1. Conditions $\mathrm{H} 1, \mathrm{H} 2$ and $\mathrm{H} 4$ are satisfied.

2. $\lim \sup _{n \rightarrow \infty} \int_{n}^{n+1} p(t) d t=\infty$. 
3. If $u \in \mathcal{D}_{t_{0}}$, then $\lim _{n \rightarrow \infty} u(n) /(\mathcal{A} u)(n)<\infty$.

Then all regular solutions of the equation (2) oscillate.

Proof. Suppose that $x(t)$ is a nonoscillating solution of (2). Without loss of generality we can assume that $x(t)>0$ in $\left[N_{x}, \infty\right), N_{x} \geq t_{0}, N_{x}$ is an integer. From $\mathrm{H} 4$ it follows that there exists an integer $N_{\mathcal{A} x} \geq N_{x}$ such that $(\mathcal{A} x)(t)>0$ for $t \geq N_{\mathcal{A} x}$. Let $N$ be an integer, $N \geq N_{\mathcal{A} x}$. Integrate (2) from $N$ to $N+1$ and obtain

$$
x(N+1)-x(N)=-\int_{N}^{N+1} p(t)(\mathcal{A} x)([t]) d t=-(\mathcal{A} x)(N) \int_{N}^{N+1} p(t) d t .
$$

But $-x(N)<x(N+1)-x(N)$. Hence $x(N)>(\mathcal{A} x)(N) \int_{N}^{N+1} p(t) d t$, i.e.,

$$
\limsup _{N \rightarrow \infty} \int_{N}^{N+1} p(t) d t=\lim _{N \rightarrow \infty} \frac{x(N)}{(\mathcal{A} x)(N)}<\infty
$$

which contradicts condition 2 .

THEOREM 6. Let the following conditions hold:

1. Conditions $\mathrm{H} 1, \mathrm{H} 2$ and $\mathrm{H} 4$ are satisfied.

2. $p(t) \geq 0$ for $t \geq t_{0}$.

3. $h \in L_{\mathrm{loc}}\left(\left[t_{0}, \infty\right) ; \mathbb{R}\right)$ and

$$
\liminf _{t \rightarrow \infty} \int_{t_{0}}^{t} h(s) d s=-\infty, \quad \limsup _{t \rightarrow \infty} \int_{t_{0}}^{t} h(s) d s=\infty .
$$

Then all regular solutions of the equation (3) oscillate.

Proof. Suppose that $x(t)$ is a nonoscillating solution of (3). Assume that $x(t)>0$ for $t \geq N$, where $N \geq t_{0}$ is an integer. Integrate (3) from $N$ to $t(t>N)$ and obtain

$$
x(t)=x(N)+\int_{N}^{t} h(s) d s-\int_{N}^{t} p(s)(\mathcal{A} x)([s]) d s \leq x(N)+\int_{N}^{t} h(s) d s .
$$

Hence $\liminf _{t \rightarrow \infty} x(t)<0$, which contradicts the assumption that $x(t)$ is eventually positive.

\section{Some particular realizations of the operator $\mathcal{A}$}

Corollary 1. Let the following conditions hold:

1. $(\mathcal{A} x)(t)=\max _{s \in M(t)} x(s)$, where $M(t)=\left[p_{1}(t), q_{1}(t)\right]$ is a compact subset of $\left[t_{0}, \infty\right)$ for $t \geq t_{0}$ and $p_{1}(t)<q_{1}(t)$ for $t \geq t_{0}, p_{1}, q_{1} \in$ $C\left(\left[t_{0}, \infty\right) ; \mathbb{R}\right), \lim _{t \rightarrow \infty} p_{1}(t)=\infty$.

2. Condition H1 and condition 2 of Theorem 1 are satisfied. 
Then all regular solutions $x(t)$ of the equation

$$
x^{\prime}(t)+p(t) \max _{s \in M([t])} x(s)=0
$$

are nonoscillating.

Proof. It is immediately verified that condition 1 implies $\mathrm{H} 3$ and $\mathrm{H} 4$. Condition H2 follows from Lemma 1 of [2]. Thus Corollary 1 follows from Theorem 1.

COROLLARY 2. Let the following conditions hold: lary 1.

1. $(\mathcal{A} x)(t)=\min _{s \in M(t)} x(s)$, where $M(t)$ is as in condition 1 of Corol-

2. Condition $\mathrm{H} 1$ and condition 2 of Theorem 1 are satisfied.

Then all regular solutions $x(t)$ of the equation

$$
x^{\prime}(t)+p(t) \min _{s \in M([t])} x(s)=0
$$

are nonoscillating.

Proof. It is immediately verified that condition 1 implies $\mathrm{H} 3, \mathrm{H} 4$ and condition 3 of Theorem 2. Condition H2 follows from Lemma 1 of [2]. Thus Corollary 2 follows from Theorem 2.

Corollary 3. Let the following conditions hold:

1. Condition 1 of Corollary 2 is satisfied.

2. Condition $\mathrm{H} 1$, condition 3 of Theorem 2 and condition 2 of Theorem 3 are satisfied.

Then each bounded solution of the equation (4) is nonoscillating.

Proof. Apply Corollary 2 and Theorem 3.

Corollary 4. Let the following conditions hold:

1. $(\mathcal{A} x)(t)=\int_{t-a}^{t} k(t, s) x(s) d s$, where $a$ is a positive constant and $k \in$ $C\left(\left[t_{0}+a\right)^{2} ;(0, \infty)\right)$.

2. Condition 2 of Corollary 1 is satisfied.

Then all regular solutions $x(t)$ of the equation

$$
x^{\prime}(t)+p(t) \int_{[t]-a}^{[t]} k([t], s) x(s) d s=0
$$

are nonoscillating.

Proof. This follows from Theorem 1. 
EXAmple 1. Consider the differential equation

$$
x^{\prime}(t)-\frac{1}{a} e^{t-[t]} \int_{[t]-a}^{[t]} e^{[t]-s} x(s) d s=0,
$$

where $a=$ const $>0$ and $t \geq t_{0}>a+2$. Here the functions

$$
p(t)=-\frac{1}{a} e^{t-[t]}, \quad(\mathcal{A} x)(t)=\int_{t-a}^{t} e^{t-s} x(s) d s
$$

satisfy the conditions of Corollary 4. Thus all solutions of the equation (5) are nonoscillating.

COROLlary 5. Let the following conditions hold:

1. $(\mathcal{A} x)(t)=f(x(g(t)))$, where $g \in C\left(\left[t_{1}, \infty\right) ; \mathbb{R}\right)$ and $t_{1} \geq t_{0}$ is such that $g(t) \geq t_{0}$ for $t \geq t_{1}, \lim _{t \rightarrow \infty} g(t)=\infty, f \in C(\mathbb{R} ; \mathbb{R}), u f(u)>0, f(0)=0$.

2. Condition 2 of Corollary 1 is satisfied.

Then all regular solutions $x(t)$ of the equation

$$
x^{\prime}(t)+p(t) f(x(g([t])))=0
$$

are nonoscillating.

Proof. This follows from Theorem 1.

EXAmple 2. Consider the differential equation

$$
x^{\prime}(t)-e^{t-3[t]} x^{3}([t])=0, \quad t \geq t_{0}>0 .
$$

Here the functions $f(u)=u^{3}, p(t)=-e^{t-3[t]}$, and $(\mathcal{A} x)(t)=x(t)$ satisfy the conditions of Corollary 5. Thus all solutions of the equation (6) are nonoscillating.

COROllary 6. Let the following conditions hold:

1. Condition 1 of Corollary 4 holds.

2. Conditions 2 and 3 of Theorem 6 hold.

Then all solutions of the equation

$$
x^{\prime}(t)+p(t) \int_{[t]-a}^{[t]} k([t], s) x(s) d s=h(t)
$$

are nonoscillating.

Proof. This follows from Theorem 6 since it is immediately verified that the corresponding operator $\mathcal{A}$ satisfies conditions $\mathrm{H} 2$ and $\mathrm{H} 4$. 
Acknowledgements. The present investigation is supported by the Bulgarian Ministry of Education, Science and Technologies under Grant MM-422.

\section{References}

[1] A. R. Aftabizadeh and J. Wiener, Oscillatory properties of first order linear functional differential equations, Appl. Anal. 20 (1985), 165-187.

[2] V. G. Angelov and D. D. Bainov, On the functional differential equations with "maximums", ibid. 16 (1983), 187-194.

[3] S. Busenberg and K. L. Cooke, Models of vertically transmitted diseases with sequential continuous dynamics, in: Nonlinear Phenomena in Mathematical Science, V. Lakshmikantham (ed.), Academic Press, New York, 1982, 179-187.

[4] K. L. Cooke and J. Wiener, Retarded differential equations with piecewise constant delays, J. Math. Anal. Appl. 99 (1984), 265-297.

[5] B. G. Zhang and N. Parhi, Oscillatory and nonoscillatory properties of first order differential equations with piecewise constant deviating arguments, ibid. 139 (1989), $23-35$.

D. D. Bainov

Medical University of Sofia

P.O. Box 45

Sofia 1504, Bulgaria
M. B. Dimitrova

Technical University

Sliven, Bulgaria 